\title{
Applications of FIESTA
}

\section{Tentyukov*}

Institut für Theoretische Teilchenphysik, Karlsruhe Institute of Technology (KIT), D-76128

Karlsruhe, Germany

E-mail: tentukoveparticle.uni-karlsruhe.de

\section{A.V. Smirnov}

Scientific Research Computing Center, Moscow State University, 119992 Moscow, Russia E-mail: asmirnov80@gmail.com

\begin{abstract}
Sector decomposition in its practical aspect is a constructive method used to evaluate Feynman integrals numerically. We present a new program performing the sector decomposition and integrating the expression afterwards. The program can be also used in order to expand Feynman integrals automatically in limits of momenta and masses with the use of sector decompositions and Mellin-Barnes representations. The program is parallelizable on modern multicore computers and even on multiple computers. Also we demonstrate some new numerical results for four-loop massless propagator master integrals.
\end{abstract}

13th International Workshop on Advanced Computing and Analysis Techniques in Physics Research February 22-27, 2010

Jaipur, India

\footnotetext{
* Speaker.
} 


\section{Introduction}

Originally sector decomposition was used as a tool for analyzing the convergence and proving theorems on renormalization and asymptotic expansions of Feynman integrals [1, 2, 3, 4, 5]. After [6], the sector decomposition approach has become an efficient tool for numerical evaluating Feynman integrals (see Ref. [7] for a recent review). At present, there are two public codes performing the sector decomposition [8] and [9]. The latter one was developed by the present authors; it is named FIESTA which stands for "Feynman Integral Evaluation by a Sector decomposiTion Approach". Recently FIESTA was greatly improved in various aspects [10].

During the last year FIESTA was widely used, some of application are listed in [11]. In [12] we used FIESTA in order to confirm numerically the recent analytic results for master integrals (MI's) for four-loop massless propagators which recently were analytically evaluated in [13]. Here we provide some more numerical results for extra orders in epsilon expansions for these MI's.

\section{Theoretical background and software structure}

FIESTA calculates Feynman integrals with the sector decomposition approach. After performing Dirac and Lorentz algebra one is left with a scalar dimensionally regularized Feynman integral [14] $F\left(a_{1}, \ldots, a_{n}\right) \int \cdots \int \frac{\mathrm{d}^{d} k_{1} \ldots \mathrm{d}^{d} k_{l}}{E_{1}^{a_{1}} \ldots E_{n}^{a_{n}}}$, where $d=4-2 \varepsilon$ is the space-time dimension, $a_{n}$ are indices, $l$ is the number of loops and $1 / E_{n}$ are propagators. We work in Minkowski space where the standard propagators are the form $1 /\left(m^{2}-p^{2}-i 0\right)$. Other propagators are permitted, see [9]. Substituting $\frac{1}{E_{i}^{a_{i}}}=\frac{e^{a i \pi / 2}}{\Gamma(a)} \int_{0}^{\infty} \mathrm{d} \alpha \alpha^{a_{i}-1} e^{-i E_{i} \alpha}$, after usual tricks [9], performing the decomposition of the integration region into the so-called primary sectors [6] and making a variable replacement, one results in a linear combination of integrals $\int_{x_{j}=0}^{1} d x_{i} \ldots d x_{n^{\prime}}\left(\prod_{j=1}^{n^{\prime}} x_{j}^{a_{j}-1}\right) \frac{U^{A-(l+1) d / 2}}{F^{A-l d / 2}}$

If the functions $\frac{U^{A-(l+1) d / 2}}{F^{A-l d / 2}}$ had no singularities in $\varepsilon$, one would be able to perform the expansion in $\varepsilon$ and perform the numerical integration afterwards. However, in general one has to resolve the singularities first. Thus, one starts a process the sector decomposition aiming to end with a sum of similar expressions, but with new functions $U$ and $F$ which have no singularities (all the singularities are now due to the part $\prod_{j=1}^{n} x_{j}^{a_{j}^{\prime}-1}$ ). The way sector decomposition is performed is called a sector decomposition strategy ([6, 8, 9]) and is an essential part of the algorithm (let us also mention a geometrical approach to sector decomposition [15] which is rather complicated in implementation as a strategy on a computer but promises to be the optimal one).

After the sector decomposition one resolves the singularities by evaluating the first terms of the Taylor series: in those terms one integration is taken analytically. Afterwards the $\varepsilon$-expansion can be performed and finally one can do the numerical integration.

FIESTA is written in Mathematica [17] and C. The user is not supposed to use the C part directly as it is launched from Mathematica via the Mathlink protocol in order to perform a numerical integration. To run FIESTA, the user has to load the FIESTA package into Mathematica 6 or 7 . In order to evaluate a Feynman integral one has to use the command SDEvaluate [UF [loop_momenta, propagators, subst], indices, order], where loop_momenta is a list of all loop momenta, propagators is a list of all propagators, subst is a list of substitutions for external momenta, masses and other values. For example, 


$$
\begin{aligned}
& \text { SDEvaluate }\left[\mathrm { UF } \left[\{\mathrm{k}\},\left\{-\mathrm{k}^{2},-\left(\mathrm{k}+\mathrm{p}_{1}\right)^{2},-\left(\mathrm{k}+\mathrm{p}_{1}+\mathrm{p}_{2}\right)^{2},-\left(\mathrm{k}+\mathrm{p}_{1}+\mathrm{p}_{2}+\mathrm{p}_{4}\right)^{2}\right\},\right.\right. \\
& \left\{\mathrm{p}_{1}^{2} \rightarrow 0, \mathrm{p}_{2}^{2} \rightarrow 0, \mathrm{p}_{4}^{2} \rightarrow 0, \mathrm{p}_{1} \mathrm{p}_{2} \rightarrow-\mathrm{s} / 2, \mathrm{p}_{2} \mathrm{p}_{4} \rightarrow-\mathrm{t} / 2, \mathrm{p}_{1} \mathrm{p}_{4} \rightarrow-(\mathrm{s}+\mathrm{t}) / 2,\right. \\
& \mathrm{s} \rightarrow-3, \mathrm{t} \rightarrow-1\}],\{1,1,1,1\}, 0]
\end{aligned}
$$

evaluats the massless on-shell box diagram with Mandelstam variables equal to -3 and -1 .

\section{Numerical results for four-loop massless propagators}
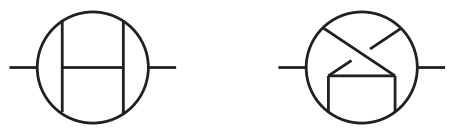

$M_{61}, \varepsilon^{1}$

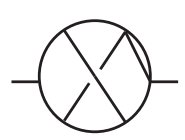

$M_{42}, \varepsilon^{1}$

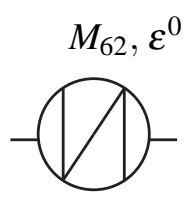

$M_{44}, \varepsilon^{0}$

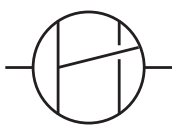

$M_{63}, \varepsilon^{0}$

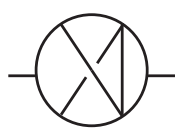

$M_{45}, \varepsilon^{1}$

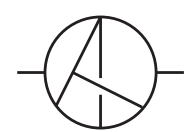

$M_{51}, \varepsilon^{1}$

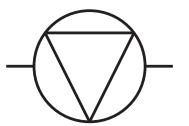

$M_{34}, \varepsilon^{3}$

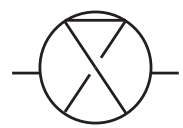

$M_{41}, \varepsilon^{1}$

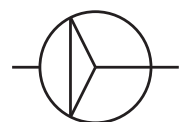

$M_{35}, \varepsilon^{2}$

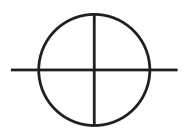

$M_{36}, \varepsilon^{1}$

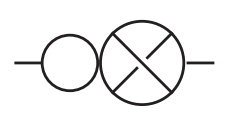

$M_{52}, \varepsilon^{1}$

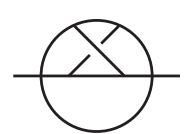

$M_{43}, \varepsilon^{1}$

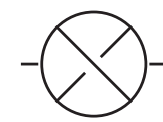

$N_{0}, \varepsilon^{2}$

Figure 1: $M_{61}-M_{43}:$ the thirteen complicated four-loop master integrals according to [13]. The two MI's $M_{52}$ and $M_{43}$ can be identically expressed through the three-loop nonplanar MI $N_{0}$.

In [18] a full set of four-loop massless propagator-like MI's was identified. There are 28 independent MI's. Analytical results for these integrals were obtained in [13]. The most complicated MI's are demonstrated on Fig. 1. $\varepsilon^{m}$ after $M_{i j}$ stands for the maximal term in $\varepsilon$-expansion of $M_{i j}$ which one needs to know for evaluation of the contribution of the integral to the final result for a four-loop integral after reduction is done, see [13]. Two of the complicated integrals $\left(M_{43}\right.$ and $\left.M_{52}\right)$ are related by a simple factor with the three-loop MI $N_{0}$ [12] so it is enough to evaluate remaining eleven complicated MI's $M_{61}-M_{36}$ as well as first three terms of the $\varepsilon$-expansion of $N_{0}$.

We calculated them (for $q^{2}=-1$ ) using FIESTA with the Cubar[16] Vegas integrator and 1500000 sampling points for integration. Our results alongside with the corresponding analytical expressions (transformed to the numerical form) from [13] look like follows ${ }^{1}$ :

$$
\begin{aligned}
M_{34} & \varepsilon^{-4}: 0.08333 \pm 0(0.08333) ; \varepsilon^{-3}: 0.916667 \pm 0.000018(0.91666) ; \varepsilon^{-2}: 5.64251 \pm 0.00022 \\
& (5.6425109) ; \varepsilon^{-1}: 27.6413 \pm 0.00077(27.6412581) ; \varepsilon^{0}: 98.638 \pm 0.0034(98.637928) ; \varepsilon^{1}: \\
& 342.736 \pm 0.012(342.7349920) ; \varepsilon^{2}: 857.88 \pm 0.048(857.8735165) ; \varepsilon^{3}: 2659.84 \pm 0.19 \\
& (2659.825402) ; \varepsilon^{4}: 4344.28 \pm 0.75 \text { (unknown); } \varepsilon^{5}: 17483.1 \pm 5.7 \text { (unknown). } \\
M_{35} & \varepsilon^{-2}: 0.601028 \pm 0.000012(0.601028) ; \varepsilon^{-1}: 7.4231 \pm 0.00024(7.423055) ; \varepsilon^{0}: 44.9127 \pm \\
& 0.00073(44.91255) ; \varepsilon^{1}: 217.023 \pm 0.0037(217.0209) ; \varepsilon^{2}: 780.436 \pm 0.013(780.432) ; \varepsilon^{3}: \\
& 2678.13 \pm 0.053 \text { (unknown);} \varepsilon^{4}: 7195.9 \pm 0.3 \text { (unknown). }
\end{aligned}
$$

\footnotetext{
${ }^{1}$ Please, note that the overall normalization used by FIESTA is different from the one employed by the authors of [13], see [12].
} 


$$
\begin{aligned}
& M_{36} \varepsilon^{-1}: 5.184645 \pm 0.000042 \text { (5.1846388); } \varepsilon^{0}: 38.8948 \pm 0.00039 \text { (38.8946741); } \varepsilon^{1}: 240.069 \\
& \pm 0.0019 \text { (240.0684359); } \varepsilon^{2}: 948.623 \pm 0.0091 \text { (unknown); } \varepsilon^{3}: 3679.77 \pm 0.06 \text { (unknown). } \\
& M_{41} \varepsilon^{-1}: 20.73860 \pm 0.00023(20.7385551) ; \varepsilon^{0}: 102.033 \pm 0.003(102.0326759) ; \varepsilon^{1}: 761.60 \pm \\
& 0.011 \text { (761.5969858); } \varepsilon^{2}: 2326.18 \pm 0.062 \text { (unknown); } \varepsilon^{3}: 12273.6 \pm 0.4 \text { (unknown). } \\
& M_{42} \varepsilon^{-1}: 20.73860 \pm 0.00024 \text { (20.7385551); } \varepsilon^{0}: 145.381 \pm 0.0029 \text { (145.3808999); } \varepsilon^{1}: 985.91 \\
& \pm 0.014 \text { (985.9082306); } \varepsilon^{2}: 3930.65 \pm 0.076 \text { (unknown); } \varepsilon^{3}: 17486.6 \pm 0.6 \text { (unknown). } \\
& M_{44} \varepsilon^{0}: 55.58537 \pm 0.00031 \text { (55.5852539); } \varepsilon^{1}: 175.325 \pm 0.004 \text { (unknown); } \varepsilon^{2}: 1496.52 \pm 0.02 \\
& M_{45} \varepsilon^{0}: 52.0181 \pm 0.0003(52.0178687) ; \varepsilon^{1}: 175.50 \pm 0.0036 \text { (175.496447); } \varepsilon^{2}: 1475.272 \pm \\
& 0.0098 \text { (unknown); } \varepsilon^{3}: 2623.5 \pm 0.1 \text { (unknown). } \\
& M_{51} \varepsilon^{-1}:-5.184651 \pm 0.000048(-5.184638) ; \varepsilon^{0}:-32.0962 \pm 0.00057 \text { (-32.09614); } \varepsilon^{1}:-91.158 \\
& \pm 0.0052 \text { (-91.1614); } \varepsilon^{2}: 119.06 \pm 0.043 \text { (unknown); } \varepsilon^{3}: 2768.6 \pm 0.45 \text { (unknown). } \\
& N_{0} \quad \varepsilon^{0}: 20.73857 \pm 0.00026 \text { (20.7385551); } \varepsilon^{1}: 190.60 \pm 0.0023 \text { (190.600238); } \varepsilon^{2}: 1049.20 \pm \\
& 0.014 \text { (1049.194196); } \varepsilon^{3}: 4423.84 \pm 0.072 \text { (unknown); } \varepsilon^{4}: 16028.8 \pm 0.5 \text { (unknown). } \\
& M_{61} \varepsilon^{-1}:-10.36931 \pm 0.00006(-10.3692776) ; \varepsilon^{0}:-70.990 \pm 0.0011(-70.99081719) ; \varepsilon^{1}:- \\
& 21.650 \pm 0.013 \text { (-21.663005); } \varepsilon^{2}: 2832.69 \pm 0.096 \text { (unknown). } \\
& M_{62} \varepsilon^{-1}:-10.36933 \pm 0.00006(-10.36927) ; \varepsilon^{0}:-58.6187 \pm 0.0013(-58.6210) ; \varepsilon^{1}: 244.681 \pm \\
& 0.015 \text { (unknown). } \\
& M_{63} \varepsilon^{-1}:-5.18467 \pm 0.000042(-5.184638) ; \varepsilon^{0}: 14.3989 \pm 0.00081 \text { (14.39739); } \varepsilon^{1}: 739.979 \pm \\
& 0.0099 \text { (unknown). }
\end{aligned}
$$

Here for each MI we provide our numerical result for coefficients of $\varepsilon$-expansion in comparison (in parentheses) with the known from [13] analiycal results (if any). As we can see, our calculations reproduce the result of [13] with 3-4 correct digits. The extra terms in the $\varepsilon$-expansion of each MI which are currently unavailable analytically but are necessary for future five-loop calculations.

\section{Conclusion}

Usually, analytical evaluation of multiloop MI is a kind of art. It requires a lot of efforts (and CPU time). In many situations, independent checkup is hardly any possible in reasonable time. That is why the simple in use tools for numerical evaluation like FIESTA are important.

Acknowledgments. This work was supported in part by DFG through SBF/TR 9 and the Russian Foundation for Basic Research through grant 08-02-01451.

\section{References}

[1] K. Hepp, Commun. Math. Phys. 2 (1966) 301. 
[2] E.R. Speer, J. Math. Phys., 9 (1968) 1404; M.C. Bergère and J.B. Zuber, Commun. Math. Phys. 35 (1974) 113; M.C. Bergère and Y.M. Lam, J. Math. Phys. 17 (1976) 1546; O.I. Zavialov, Renormalized quantum field theory, Kluwer Academic Publishers, Dodrecht (1990); V.A. Smirnov, Commun. Math. Phys. 134 (1990) 109.

[3] P. Breitenlohner and D. Maison, Commun. Math. Phys. 52 (1977) 11; 39,55;

[4] M.C. Bergère, C. de Calan and A.P.C. Malbouisson, Commun. Math. Phys. 62 (1978) 137; K. Pohlmeyer, J. Math. Phys. 23 (1982) 2511.

[5] V.A. Smirnov, Applied asymptotic expansions in momenta and masses, STMP 177, Springer, Berlin, Heidelberg (2002).

[6] T. Binoth and G. Heinrich, Nucl. Phys. B, 585 (2000) 741; Nucl. Phys. B, 680 (2004) 375; Nucl. Phys. B, 693 (2004) 134.

[7] G. Heinrich, Int. J. of Modern Phys. A, 23 (2008) 10. [arXiv:0803.4177].

[8] C. Bogner and S. Weinzierl, Comput. Phys. Commun. 178 (2008) 596 [arXiv:0709.4092 [hep-ph]]; Nucl. Phys. Proc. Suppl. 183 (2008) 256 [arXiv:0806.4307 [hep-ph]].

[9] A.V. Smirnov and M.N. Tentyukov, Comput. Phys. Commun. 180 (2009) 735 [arXiv:0807.4129 [hep-ph]].

[10] A.V. Smirnov, V.A. Smirnov and M.N. Tentyukov,[arXiv:0912.0158 [hep-ph]].

[11] A. V. Smirnov, V. A. Smirnov and M. Steinhauser, Phys. Lett. B 668, 293 (2008) [arXiv:0809.1927 [hep-ph]]; R. Bonciani and A. Ferroglia, JHEP 0811, 065 (2008) [arXiv:0809.4687 [hep-ph]]; Y. Kiyo, D. Seidel and M. Steinhauser, JHEP 0901, 038 (2009) [arXiv:0810.1597 [hep-ph]];G. Bell, Nucl. Phys. B 812, 264 (2009) [arXiv:0810.5695 [hep-ph]];V. N. Velizhanin, arXiv:0811.0607 [hep-th];T. Ueda and J. Fujimoto, arXiv:0902.2656 [hep-ph];D. Seidel, arXiv:0902.3267 [hep-ph];;G. Heinrich, T. Huber, D. A. Kosower and V. A. Smirnov, Phys. Lett. B 678, 359 (2009) [arXiv:0902.3512 [hep-ph]];P. A. Baikov, K. G. Chetyrkin, A. V. Smirnov, V. A. Smirnov and M. Steinhauser, Phys. Rev. Lett. 102, 212002 (2009) [arXiv:0902.3519 [hep-ph]];J. Gluza, K. Kajda, T. Riemann and V. Yundin, PoS A CAT08, 124 (2008) [arXiv:0902.4830 [hep-ph]];S. Bekavac, A. G. Grozin, D. Seidel and V. A. Smirnov, Nucl. Phys. B 819, 183 (2009) [arXiv:0903.4760 [hep-ph]];R. Bonciani, A. Ferroglia, T. Gehrmann and C. Studerus, JHEP 0908, 067 (2009) [arXiv:0906.3671 [hep-ph]];M. Czakon, A. Mitov and G. Sterman, Phys. Rev. D 80, 074017 (2009) [arXiv:0907.1790 [hep-ph]];A. Ferroglia, M. Neubert, B. D. Pecjak and L. L. Yang, arXiv:0907.4791 [hep-ph]; JHEP 0911, 062 (2009) [arXiv:0908.3676 [hep-ph]];S. Bekavac, A. G. Grozin, P. Marquard, J. H. Piclum, D. Seidel and M. Steinhauser, arXiv:0911.3356 [hep-ph];M. Dowling, J. Mondejar, J. H. Piclum and A. Czarnecki, arXiv:0911.4078 [hep-ph];A. V. Smirnov, V. A. Smirnov and M. Steinhauser, [arXiv:0911.4742 [hep-ph]].

[12] A.V. Smirnov, M. Tentyukov, [arXiv:1004.1149 [hep-ph]].

[13] P.A. Baikov and K.G. Chetyrkin, [arXiv:1004.1153 [hep-ph]].

[14] G. 't Hooft and M. Veltman, Nucl. Phys. B 44 (1972) 189;C.G. Bollini and J.J. Giambiagi, Nuovo Cim. 12 B (1972) 20.

[15] T. Kaneko and T. Ueda, [arXiv:0908.2897 [hep-ph]].

[16] T. Hahn, Comput. Phys. Commun. 168 (2005) 78, arXiv: hep-ph/0404043

[17] Wolfram Research, Inc., Mathematica, Version 7.0, Champaign, IL (2008).

[18] P. A. Baikov, Phys. Lett. B 634, 325 (2006) [arXiv:hep-ph/0507053]. 